\title{
Adenovirus sneak attacks during the H7N9 flu season
}

\author{
XU Jun ${ }^{1 \dagger}$, LI Chen ${ }^{1 \dagger}$, WALLINE Joseph ${ }^{2} \&$ YU XueZhong ${ }^{1 *}$ \\ ${ }^{1}$ Chinese Academy of Medical Sciences, Peking Union Medical Hospital, Beijing 100730, China; \\ ${ }^{2}$ Division of Emergency Medicine, Department of Surgery, Saint Louis University Hospital, Saint Louis, Missouri 63110, USA
}

Received June 12, 2013; accepted July 2, 2013; published online July 22, 2013

Citation: $\quad$ Xu J, Li C, Walline J, et al. Adenovirus sneak attacks during the H7N9 flu season. Sci China Life Sci, 2013, 56: 863-864, doi: 10.1007/s11427-013-4529-6

The H7N9 influenza virus made headlines recently, with outbreaks in Chinese urban centers. These infections continue a recent trend of novel virus outbreaks, such as those involving SARS coronavirus and the H1N1 influenza virus. These outbreaks have been likened to tropical storms, and like prior 'viral storms' the latest H7N9 influenza virus has aroused public panic despite the fact that H7N9 infection rates are sporadic. At the same time, other more common ones (such as adenovirus) can lead to much more likely pathology. Recently we admitted two patients with severe adenovirus pneumonias in ARDS; both of them ICU-level care, mechanical ventilation and special positioning to maintain their oxygenation.

Adenoviruses are DNA viruses with no envelope, a double-stranded DNA genome, and a 20-sided icosahedral capsid composed of 252 capsomeres. They are widely prevalent in humans, with over 100 types identified, of which more than 60 can infect humans. Adenoviruses have caused epidemics in China since the 1950s. Most recently, adenovirus type 55 caused an outbreak in China's Shanxi Province in 2006. American scientists found in 2009 that adenoviruses could be transmitted from monkeys to humans and lead to infection. In February 2012, a viral outbreak in China's Hebei Province was confirmed as adenovirus type 55 .

Unlike H7N9, adenoviruses can be spread by droplets, close personal contact or by fecal-oral routes. The general population is susceptible, in particular infants and young children, as well as the immunocompromised. Adenoviruses

$\dagger$ Contributed equally to this work

*Corresponding author (email: yxzpumch@126.com) can infect multiple organ systems, including the respiratory, gastrointestinal, urinary, and ocular system. Its incubation period is typically $4-5 \mathrm{~d}$, and clinical manifestations differ between virus types.

Adenoviral respiratory infections are common, generally mild, and self-limiting with a natural course of about 7-10 d. However, adenovirus types 7 and 55 can develop into severe pneumonias with lung consolidation (Figure 1A). Adenovirus ocular infections are often a complication of adenovirus respiratory tract infections (Figure 1B).

At present, there is no specific treatment for adenovirus infections. Symptoms of infection include fever, dry throat, cough, difficulty breathing or gastrointestinal discomfort. Therapy mainly includes non-specific antivirals, early low-dose glucocorticoids, prophylactic use of antibiotics, and organ support therapy. The lack of targeted antiviral therapy makes the prevention of adenovirus infection extremely important. The strict isolation of confirmed and suspected cases, as well as maintaining rigorous personal
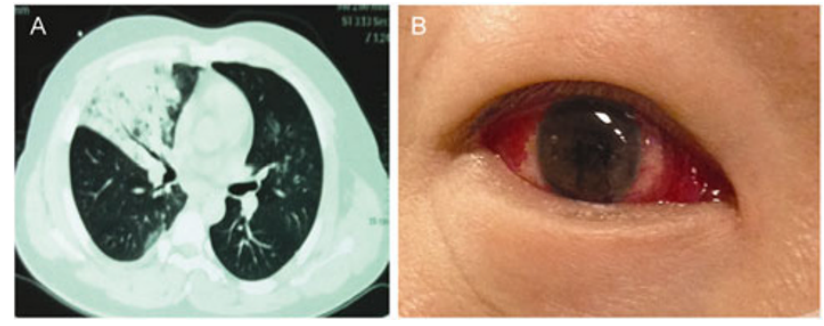

Figure 1 CT scan of adenovirus type 7 pneumonia with lung consolidation (A) and conjunctivitis (right eye) in a doctor accidentally infected by droplets from an adenovirus-infected patient (B). 
and environmental hygiene will help to enhance resistance. Although an adenovirus vaccine containing formaldehydeinactivated virus has been developed, it has not yet been widely used because of its apparent carcinogenicity in animal test subjects.

The infectivity and virulence of adenoviruses are high, but can be controlled. Hospitalization is often required for those with severe adenovirus infections. All medical institutions should take precautions against this virus, and maintain a high degree of vigilance for infections, especially when other viruses such as H7N9 are commanding so much attention.

Open Access This article is distributed under the terms of the Creative Commons Attribution License which permits any use, distribution, and reproduction in any medium, provided the original author(s) and source are credited. 